\title{
Study of the cyclotron feature in MXB 0656-072
}

\author{
V. A. McBride ${ }^{1}$, J. Wilms ${ }^{2}$, M. J. Coe ${ }^{1}$, I. Kreykenbohm ${ }^{3,4}$, R. E. Rothschild ${ }^{5}$, \\ W. Coburn ${ }^{6}$, J. L. Galache ${ }^{1}$, P. Kretschmar ${ }^{7}$, W. R. T. Edge ${ }^{1}$, and R. Staubert ${ }^{3}$
}

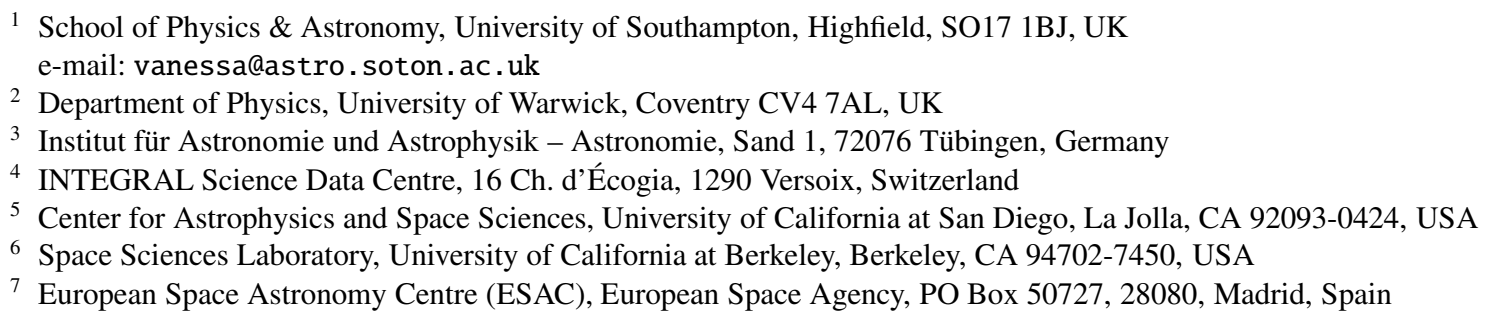

Received 22 September 2005 / Accepted 26 January 2006

\section{ABSTRACT}

We have monitored a type II outburst of the Be/X-ray binary MXB 0656-072 in a series of pointed RXTE observations during October through December 2003. The source spectrum shows a cyclotron resonance scattering feature at $32.8_{-0.4}^{+0.5} \mathrm{keV}$, corresponding to a magnetic field strength of $3.67_{-0.04}^{+0.06} \times 10^{12} \mathrm{G}$ and is stable through the outburst and over the pulsar spin phase. The pulsar, with an average pulse period of $160.4 \pm 0.4 \mathrm{~s}$, shows a spin-up of $0.45 \mathrm{~s}$ over the duration of the outburst. From optical data, the source distance is estimated to be $3.9 \pm 0.1 \mathrm{kpc}$ and this is used to estimate the X-ray luminosity and a theoretical prediction of the pulsar spin-up during the outburst.

Key words. X-rays: stars - stars: magnetic fields - stars: pulsars: individual: MXB 0656-072

\section{Introduction}

MXB 0656-072 was first classified as a transient source by Clark et al. (1975) when detected at $80 \mathrm{mCrab}$ on 1975 September 20 by SAS-3. Subsequently Ariel $V$ observed the source at 50 and $70 \mathrm{mCrab}$ on 1976 March 19 and 27 respectively (Kaluzienski 1976). Kaluzienski's comments that MXB 0656-072 may more closely resemble a long term variable source as opposed to a transient possibly led to it being classified as a Low Mass X-ray Binary in the catalogue of Liu et al. (2001).

The source, which has been dormant and unobserved since $1975 / 1976$, made a reappearance in the X-ray sky in a large, extended outburst during 2003 October. During this outburst,which lasted over 2 months and reached an X-ray luminosity of $200 \mathrm{mCrab}$, it was identified as a pulsating X-ray binary with a pulse period of $160.7 \mathrm{~s}$ (Morgan et al. 2003) and with an optical counterpart of spectral type 09.7Ve (Pakull et al. 2003), re-categorising it as a High Mass X-ray Binary. Time-resolved $B$ and $R$ photometry (Bartolini et al. 2005) of the optical counterpart during this outburst revealed no optical periodicity at the X-ray pulse period. Orbital parameters of the system remain undetermined.

Preliminary analysis of the phase averaged spectrum by Heindl et al. (2003) showed that the continuum could be fit with a power law with an exponential cutoff. A cyclotron resonant scattering feature with a centroid energy of $36 \pm 1 \mathrm{keV}$ was discovered by Heindl et al. (2003).

The strong magnetic fields found near the polar caps of neutron stars quantise the ambient electron energies into Landau levels. Photons at these resonant electron energies are scattered, creating cyclotron resonant scattering features (CRSFs) in the observed spectrum. The energy of the fundamental CRSF is approximated by:

$E_{\mathrm{C}} \simeq 11.6 \mathrm{keV} \times \frac{1}{1+z} \times \frac{B}{10^{12} \mathrm{G}}$

where $z$ is the gravitational redshift and $B$ the magnetic field strength. Thus measuring the energy of cyclotron features in the spectra of accreting X-ray binaries gives us a direct measurement of the magnetic field of the neutron star.

\section{Data reduction}

MXB 0656-072 was observed with RXTE during 2003 October through 2004 January. Observations coincided with a Type II (Stella et al. 1986) X-ray outburst and data were accumulated during both the rise and decline of the outburst. In total, the PCA livetime for this set of observations amounts to $95 \mathrm{ks}$. Figure 1 shows the MXB 0656-072 lightcurve from the All Sky Monitor (ASM) aboard RXTE 


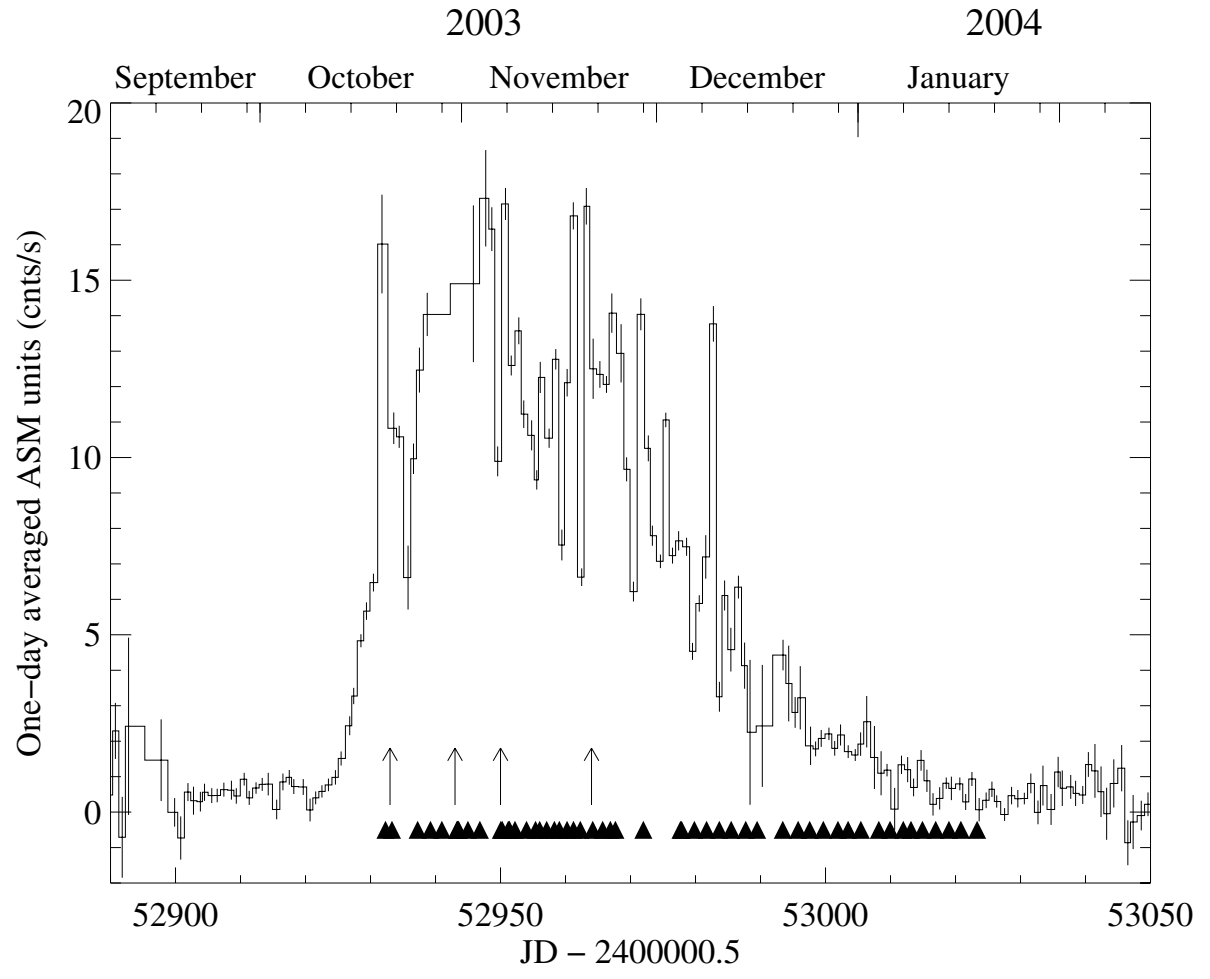

Fig. 1. The ASM lightcurve of MXB 0656-072 during the 2003 outburst with bin sizes of 1 day. Triangles indicate the dates of pointed RXTE observations. The arrows indicate the dates at which spectra were compared with each other in order to observe any spectral evolution over the outburst. This is further discussed in Sect. 3.2. during the outburst. Triangles indicate the dates of pointed RXTE observations.

While Standard $2 f$ mode $P C A$ data (with 16 s time resolution and 128 channel energy resolution) were employed to generate phase averaged spectra, the phase resolved spectra and timing results were obtained from observation-specific binnedmode data with a temporal resolution of $250 \mathrm{~ms}$ and an energy resolution of 128 channels.

Analysis was performed with HEASOFT version 5.3.1 and spectral fitting with XSPEC version 11.3.1t. Phase resolved spectra were created using a modified version of the FTOOL fasebin developed at the University of Tübingen (Kreykenbohm 2004).

For spectral fitting, PCA data (Jahoda et al. 2006) in the energy range 3-22 keV were used, to which we added systematic errors of $0.5 \%$. Below and above this range the systematic errors required are larger to account for the uncertainties in the $P C A$ response matrix, so we ignored data outside the $3-22 \mathrm{keV}$ range.

For all spectra we used the E_8 $\mu$ __256_DX1F mode HEXTE data (Rothschild et al. 1998), which have a temporal resolution of $8 \mu \mathrm{s}$ and covers the range $0-250 \mathrm{keV}$ with 256 spectral channels. Electronic thresholds limit the HEXTE energy range to $15-250 \mathrm{keV}$. In all cases, the signal-to-noise ratio was increased by adding together the data from both clusters and generating a new response file with weights 1:0.75 to account for the loss of signal from one of the detectors in the second HEXTE cluster. A binning factor of 3 was applied to data in the 70-200 keV range to improve statistical significance of model fitting while still maintaining a reasonable energy resolution. Data above $200 \mathrm{keV}$ were not used.

\section{Phase averaged analysis}

For the phase averaged spectral analysis, the spectra from all observations through the peak of the outburst (from MJD 52932 to MJD 52964) were added together in an effort to maximise the signal-to-noise ratio.

\subsection{Spectral model}

In accreting X-ray pulsars the highly ionised accretion stream is channelled onto one or both of the magnetic poles of the neutron star by its strong $\left(B \sim 10^{12} \mathrm{G}\right)$ magnetic field. Depending on the accretion rate and the strength of the magnetic field, an accretion shock may form. The continuum spectral shape is dominated by Comptonisation of photons by the accretion stream at the magnetic poles. As a comprehensive theoretical spectral model is unavailable, the spectrum is generally modelled as a exponentially cutoff power law, but see Tanaka (1986) for the choice of a Fermi-Dirac Cut Off (FDCO) model or Mihara (1995) for the NPEX model. In addition to this continuum we may observe photoelectric absorption by the accretion stream or stellar wind, an $\mathrm{Fe} \mathrm{K} \alpha$ fluorescence line and one or more cyclotron resonance scattering features.

In our analysis we describe the continuum using a power law with photon index $\Gamma$, with a high energy cutoff at $E_{\text {cut }}$.

$I_{\text {cont }}(E)=K E^{-\Gamma} \times \begin{cases}1 & \left(E \leq E_{\text {cut }}\right) \\ \mathrm{e}^{-\left(E-E_{\text {cut }}\right) / E_{\text {fold }}} & \left(E>E_{\text {cut }}\right) .\end{cases}$

The energy cutoff is smoothed by including a Gaussian absorption line (gsmooth in XSPEC) with an energy dependent width, at the continuum cutoff energy. Without a smoothing function the energy cutoff forms a discontinuity in the model and this results in residuals which can easily be mistaken as arising from 
Table 1. Spectral parameters (as defined in the text) for the phase averaged spectrum. The model consists of power law with a smoothed high energy cutoff, photoelectric absorption, a CYCLABS cyclotron feature, a Gaussian $\mathrm{Fe}$ line and a blackbody component. The flux in the $\mathrm{Fe}$ emission line is represented by $a_{\mathrm{Fe}}$, in photons $\mathrm{cm}^{-2} \mathrm{~s}^{-1}$. Uncertainties are at $90 \%$ confidence intervals.

\begin{tabular}{ll}
\hline \hline Parameter & Value \\
\hline$N_{\mathrm{H}}$ & $7.28 \times 10^{21} \mathrm{~cm}^{-2}$ \\
$\Gamma$ & $0.89_{-0.05}^{+0.07}$ \\
$E_{\text {cut }}$ & $15.5_{-0.2}^{+0.2} \mathrm{keV}$ \\
$E_{\text {fold }}$ & $11.8_{-0.3}^{+0.2} \mathrm{keV}$ \\
$T$ & $0.86_{-0.14}^{+0.08} \mathrm{keV}$ \\
$E_{\mathrm{c}}$ & $32.8_{-0.4}^{+0.5} \mathrm{keV}$ \\
$W_{\mathrm{c}}$ & $11.8_{-1.1}^{+1.0} \mathrm{keV}$ \\
$D_{\mathrm{c}}$ & $0.38_{-0.02}^{+0.02}$ \\
$E_{\mathrm{Fe}}$ & $6.48_{-0.03}^{+0.03} \mathrm{keV}$ \\
$\sigma_{\mathrm{Fe}}$ & $0.36_{-0.06}^{+0.06} \mathrm{keV}$ \\
$a_{\mathrm{Fe}}$ & $0.0195_{-0.001}^{+0.001}$ \\
\hline
\end{tabular}

a cyclotron feature (Kretschmar et al. 1997; Coburn 2001). Although we attempted to use the FDCO and NPEX models to describe the continuum, we found that the best-fit continuum was indeed a power law with a smoothed high-energy cutoff.

The spectral shape is insensitive to the H I column density and in order to include some estimate of the column density a value of $7.28 \times 10^{21} \mathrm{~cm}^{-2}$ was estimated over a cone of radius $1^{\circ}$ in the direction of the source (Dickey \& Lockman 1990). The column density, $N_{\mathrm{H}}$, was fixed at this value for subsequent spectral fitting.

Modifying the continuum is a CRSF modelled with the CYCLABS model, which has the analytical form shown below:

$C Y C L A B S(E)=D_{\mathrm{c}} \frac{\left(W_{\mathrm{c}} E / E_{\mathrm{c}}\right)^{2}}{\left(E-E_{\mathrm{c}}\right)^{2}+W_{\mathrm{c}}^{2}}$

where $E_{\mathrm{c}}, D_{\mathrm{c}}$ and $W_{\mathrm{c}}$ are the cyclotron energy, depth and width respectively (Makishima et al. 1990; Mihara et al. 1990). Using other continuum models (FDCO and NPEX) did not alter the presence of the cyclotron feature.

We model a strong $\mathrm{Fe} \mathrm{K} \alpha$ fluorescence feature with a Gaussian emission line and determine the equivalent width to be $3.66 \mathrm{keV}$. In addition we include a blackbody component to account for the excess flux at the soft end of the spectrum. The blackbody component has a temperature of $0.86_{-0.14}^{+0.08} \mathrm{keV}$ and can be attributed to emission from an accreting polar cap, in a manner similar to Coburn et al. (2001) in the case of X Per.

The fit to the phase averaged spectrum results in $\chi_{\text {red }}^{2}=1.1$ with the spectral parameters given in Table 1 . The spectrum is shown in Fig. 2.

The cyclotron feature at $32.8_{-0.4}^{+0.5} \mathrm{keV}$ is clearly visible in the residuals. A harmonic with width $16_{-14}^{+20} \mathrm{keV}$ and depth $0.19_{-0.16}^{+0.26}$ may be present at twice the fundamental energy, but detection is marginal, as can be interpreted from the uncertainties in the line parameters. Assuming that the cyclotron line at $32.8 \mathrm{keV}$ is the fundamental $(n=1)$ and a gravitational redshift of $z=0.3$ for a typical neutron star mass of $1.4 M_{\odot}$ and radius of $10 \mathrm{~km}$, we can estimate the magnetic field from Eq. (1) to be $3.67_{-0.04}^{+0.06} \times 10^{12} \mathrm{G}$.

Note the systematic feature in the residuals at $\sim 10 \mathrm{keV}$. This feature is evident in the spectra of a number of accreting X-ray binaries (GS 1843+00, Her X-1) (Coburn 2001). It has been observed in spectra accumulated by Ginga (Mihara 1995) and BeppoSAX (Santangelo et al. 1998) as well, and should be accounted for in future continuum models of accreting X-ray pulsars. A more comprehensive review of this feature can be found in Coburn (2001).

\subsection{Spectral evolution over outburst}

In order to determine whether there was any significant change in the spectral shape or the cyclotron feature during the outburst we compared the spectral parameters at various dates through the outburst. These dates are indicated by the arrows in Fig. 1 and further information is shown in Table 2 .

Note that the fourth spectrum is obtained by adding observations over a five day range in order to have signal-tonoise ratios similar to those of the first 3 datasets. We find that, on average, the spectral fits have $\chi_{\text {red }}^{2} \sim 1.2$, except for 2003 November $16-20$ which has $\chi_{\text {red }}^{2}=1.7$. The power law index stays between 0.9 and 1.1 during this time and there is very little change in $E_{\text {cut }}$ and $E_{\text {fold }}$, which have values close to those given in Table 1 . The cyclotron parameters over this time interval are shown in Fig. 3. Noting that the error bars represent $90 \%$ uncertainty levels it is clear that the cyclotron feature is stable over the outburst.

\section{Phase resolved analysis}

If the rotation and magnetic axes of the neutron star are not aligned, the observer sees X-ray emission modulated by the pulsar spin period. Due to the fact that cyclotron features are strongly dependent on viewing angle, phase resolved spectroscopy is a tool well-suited to studying cyclotron features.

Pulse profiles and phase resolved spectra were generated by performing a barycentric correction of the bin times for the PCA data and the photon arrival times for the HEXTE data for two $15 \mathrm{ks}$ observations at the peak of the outburst. Orbital modulation was not accounted for as the orbital ephemeris of the system is still unknown. The pulse period was determined by running a Lomb-Scargle analysis (Press \& Rybicki 1989; Lomb 1976; Scargle 1982) on the barycentre-corrected lightcurves. The lightcurves were then folded at the pulse period of $160.4 \mathrm{~s}$ to produce the pulse profiles shown in Fig. 4.

\subsection{Pulse profiles}

The pulse profiles are single-peaked and sinusoidal through all energy bands and show the pulsed fraction $\left(\left(F_{\max }-F_{\min }\right) / F_{\max }\right)$ increasing steadily from $26 \%$ in the $2-5 \mathrm{keV}$ band up to $40 \%$ in the $30-60 \mathrm{keV}$ band. The pulse shape is consistent with what one would expect from viewing just a single magnetic pole radiating isotropically as a blackbody, including the effects of gravitational bending around the neutron star 


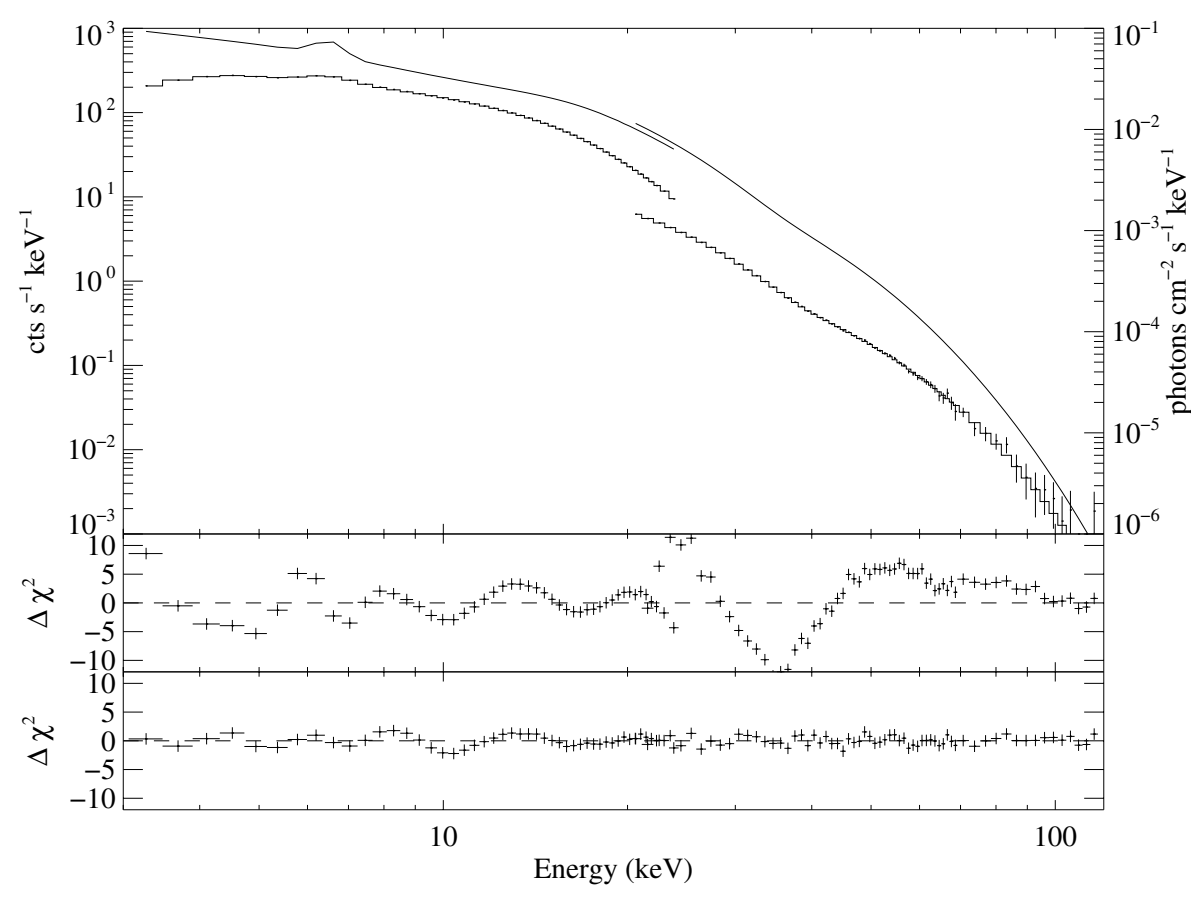

Fig. 2. The spectrum of MXB 0656-072 from 3-120 keV. The crosses show the data, the smooth curve shows the unfolded spectrum and the histogram the model fit. In the second panel the residuals, plotted as $\Delta \chi^{2}$, are shown for the case in which no CRSF is included in the model. In the lower panel, these residuals are shown again - this time including a CRSF in the model.
Table 2. Dates and $P C A$ livetimes of observations used to determine the spectral evolution over the outburst.

\begin{tabular}{lll}
\hline \hline Date & MJD & Livetime (ks) \\
\hline $2003-10-20$ & 52933 & 11 \\
$2003-10-30$ & 52943 & 8 \\
$2003-11-06$ & 52950 & 15 \\
$2003-11-(16-20)$ & $5296(0-4)$ & 7 \\
\hline
\end{tabular}

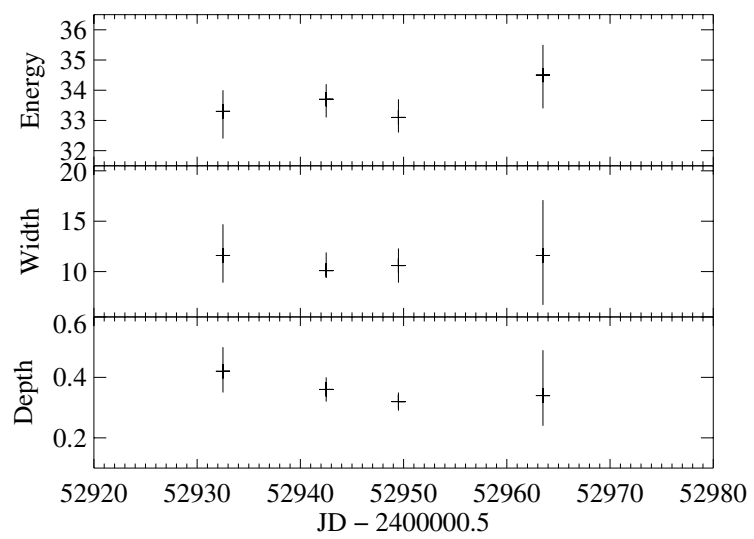

Fig. 3. Cyclotron line parameters throughout the 2003 October outburst. The energy and width are in keV.

(Beloborodov 2002). The almost perfectly sinusoidal pulse shapes may indicate that there is no absorption along the accretion column, as it is believed that strong absorption along the accretion column contributes to the complexity of the pulse profile at low energies (Kreykenbohm et al. 2002; Nagase et al. 1983). The pulse profile shape and amplitude support the conclusion reached by Bulik et al. (2003): that the rotation and magnetic axes of accreting pulsars are almost aligned.

\subsection{Spectral evolution over Pulse}

The data were split into 5 phase bins for analysis, as shown in the topmost panel of Fig. 4. This gives us good statistics at a temporal resolution sufficient to describe this particular pulse shape. Evolution of the spectral parameters with phase is shown in Fig. 5.

For the phase resolved spectra, we used the same model as in Sect. 3.1, however the addition of a blackbody component did not significantly improve the fit, so was not included. Furthermore, a Gaussian absorption component at $\sim 10 \mathrm{keV}$ was required to take account of the systematic feature at this wavelength, mentioned in Sect. 3.1.

The cyclotron feature at energy around $32 \mathrm{keV}$ is present through all pulse phases. There are, however, variations in the cyclotron line width. In general, the line is at its narrowest $\left(6_{-5}^{+3} \mathrm{keV}\right)$ in the peak rise and reaches its broadest $\left(14_{-3}^{+5} \mathrm{keV}\right)$ during the pulse decline (see Fig. 5.) The cyclotron line depth also shows some variation through phase, in a manner inverse to the continuum power law normalisation coefficient. However, the cyclotron feature in this source shows little variability with phase in comparison with some other sources such as Vela X-1 (Kreykenbohm et al. 2002) and GX 301-2 (Kreykenbohm et al. 2004), which show cyclotron line energy variations of a couple keV throughout the phase.

\section{Discussion}

\subsection{Source distance}

As the optical counterpart to MXB 0656-072 has been identified, we can use the optical magnitude and colour to estimate the distance to the source.

The optical counterpart has been identified as an 09.7Ve spectral type star, with an observed $B-V$ colour in the range 0.86 to 1.02 (Pakull et al. 2003) and an observed 


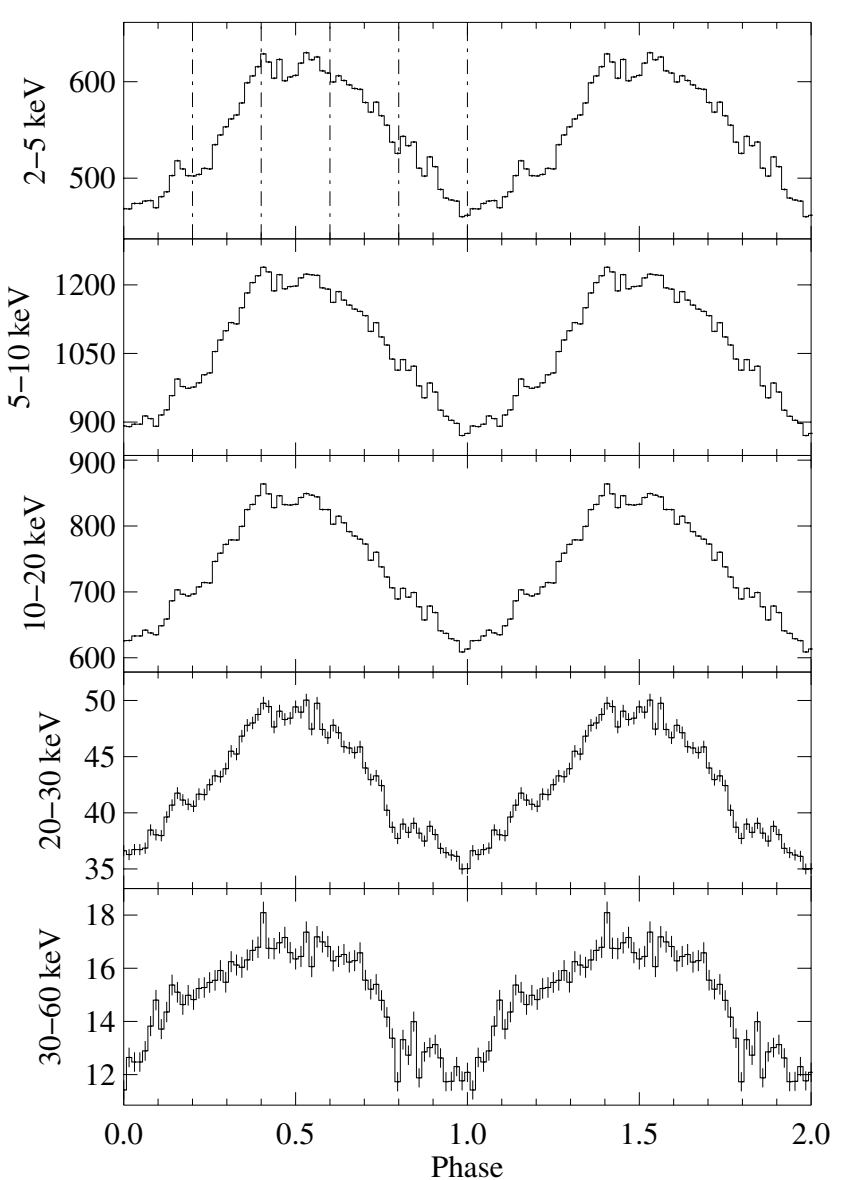

Fig. 4. Pulse profile shown in counts $\mathrm{s}^{-1}$. Profiles are plotted twice for clarity.

$V$-magnitude in the range $12.05 \mathrm{mag}$ to $12.38 \mathrm{mag}$. For this estimate we shall assume that the variability in the $V$-magnitude and the $B-V$ colour is due to the circumstellar disk contributing the the $V$-magnitude. Hence we will use the faintest available $V$-magnitude value, assuming that this is when the disk is smallest, along with the corresponding bluest $B-V$ colour. We have estimated errors typical for photometry of a 12th magnitude star on a $1-2 \mathrm{~m}$ telescope and use $V=12.38 \pm 0.02 \mathrm{mag}$ and $B-V=0.86 \pm 0.03$.

Interstellar reddening is given by:

$E(B-V)=(B-V)-(B-V)_{0}$

where $(B-V)_{0}$ is the intrinsic colour. Thus for an $09.7 \mathrm{Ve}$ star we expect $(B-V)_{0}=-0.28$ (Wegner 1994) and an absolute magnitude of $M_{V}=-4.2 \mathrm{mag}$ (Zombeck 1990).

This puts the magnitude of reddening in the $V$-band at $A=$ $3.7 \pm 0.1 \mathrm{mag}$. Knowing the apparent magnitude, the absolute magnitude and the reddening magnitude allows us to estimate the distance using:

$D=10^{1+(m-M-A) / 5}=3.9 \pm 0.1 \mathrm{kpc}$.

It is most likely that the optical colour and magnitude used in this calculation were observed at time when the contribution from the Be star disk was not negligible. Hence the above distance estimate will be an underestimate.

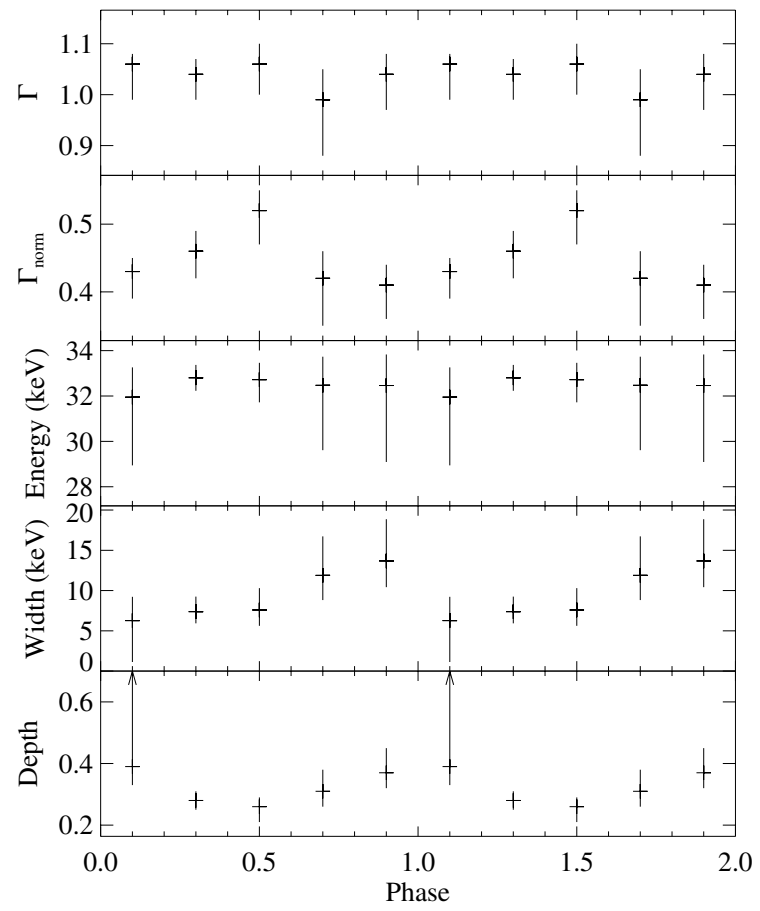

Fig. 5. From top to bottom the power law index, the power law normalisation, the cyclotron energy, cyclotron line width and cyclotron line depth are plotted as a function of phase. Error bars indicate $90 \%$ uncertainty levels.

\subsection{Spin-up trends}

Accretion of material onto a pulsar is expected to transfer a significant amount of angular momentum to the neutron star. Thus an accreting X-ray pulsar undergoing an outburst is in a state where the mass (and thus momentum) transfer rate is high and one expects to observe a slight decrease in the pulse period as it is spun-up.

We have analysed all pointed $P C A$ data in the $2-20 \mathrm{keV}$ energy range and determined pulse periods as a function of time. Initially, to derive the pulse period, we used a LombScargle analysis (Press \& Rybicki 1989). Since the resulting values had a rather high uncertainty, the method of phase connection (Deeter et al. 1981; Muno et al. 2002) was applied in a second step: average pulse profiles (with an initial period of $P=160.4$ s) for data sets of individual RXTE orbits (of typical length 1 to $3 \mathrm{ks}$ ) were produced and phase connected by an integer number of pulse cycles. Using an improved solution for the pulse ephemeris, including $\dot{P}$, also the larger gaps between data sets could be uniquely bridged. The result is shown in Fig. 6. The measurements are consistent with an initial period of $160.58 \mathrm{~s}$ at MJD 52930 and an average spin-up until MJD 52971 of $\dot{P}=-0.0101 \pm 0.0003 \mathrm{~s} \mathrm{day}^{-1}$.

After MJD 52971 the accretion rate drops to a level low enough to halt spin-up of the neutron star. This is evidenced by a flattening of the slope in the pulse period vs time plot shown in Fig. 6. The data after MJD 52971 are consistent with a constant period. This levelling off of the pulse period to a constant value after MJD 52971 indicates that the pulse period changes are an effect of accretion torques rather than modulation by the orbital period of the neuton star. 


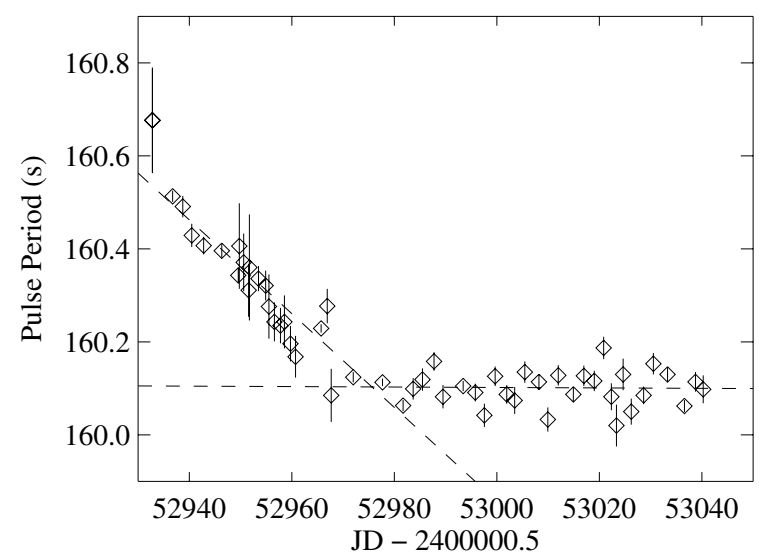

Fig. 6. Pulse period as a function of time through the outburst, with a linear fit to the data.

From the average $\mathrm{X}$-ray flux in the $2-10 \mathrm{keV}$ band $(4.35 \times$ $10^{-9} \mathrm{erg} \mathrm{cm}^{-2} \mathrm{~s}^{-1}$ ) we can calculate the X-ray luminosity if the distance to the source is known. Using our estimate of $3.9 \pm 0.1 \mathrm{kpc}$ for the source distance from Sect. 5.1, the X-ray luminosity of the source is $6.6 \pm 0.4 \times 10^{36} \mathrm{erg} \mathrm{s}^{-1}$. Then a straightforward application of the accretion torque model by Ghosh \& Lamb (1979) (Eq. (15) with standard neutron star parameters and our own values of $B=3.67 \times 10^{12} \mathrm{G}$ and $L_{\mathrm{x}}=$ $6.6 \times 10^{36} \mathrm{erg} \mathrm{s}^{-1}$ ) yields a spin-up rate of $\dot{P}=-0.0034 \mathrm{~s} \mathrm{day}^{-1}$. This is about a factor of three smaller than the observed value. We do not consider this a problem, as accreting binary pulsars very generally show a broad range of behaviour with respect to the development of their spin periods with time (for reviews see Nagase 1989 and Bildsten et al. 1997). This includes episodes of spin-up and spin-down, depending on the exact conditions of accretion at the time. For a transient in outburst such as MXB 0656-072 one would expect to observe a strong spin-up, since under the greatly increased mass accretion rate, as evidenced by the large increase in X-ray luminosity, the system will tend to be far from any equilibrium condition.

\section{Summary}

From analysis of the pointed RXTE observations of MXB 0656-072 and limited optical data available we can deduce the following:

- The X-ray spectrum continuum is best fit by a power law model with an exponential cutoff, where a Gaussian function with an energy dependent width is used to smooth discontinuities at the cutoff energy.

- A cyclotron resonance scattering feature is present at an energy of $32.8_{-0.5}^{+0.4} \mathrm{keV}$ and the energy is independent of the continuum model used to describe the spectrum. The CRSF is present at this energy in all observations throughout the outburst. The CRSF is present throughout the entire neutron star spin phase, but the width and depth of the feature show variation through the spin phase.

- Although there is no convincing evidence for higher harmonic cyclotron lines in this data, the possibility of a higher harmonic cannot be ruled out.
- The magnetic field inferred from the cyclotron energy (by assuming that the detected line is the fundamental CRSF) is $3.67_{-0.06}^{+0.04} \times 10^{12} \mathrm{G}$.

- On the basis of optical photometric data, the distance to MXB 0656-072 is estimated to be $3.9 \pm 0.1 \mathrm{kpc}$.

- Over the time considered, we have measured a strong spinup (about $0.45 \mathrm{~s}$ in 30 days), which appears quite reasonable under the conditions observed in this high mass X-ray binary in outburst.

Acknowledgements. V.A.M. would like to acknowledge the NRF(S.Africa), the British Council and Southampton University. I.K. acknowledges DLR grants 50 OG 9601 and 50 OG 0501.

\section{References}

Bartolini, C., Casares, J. V., Guarnieri, A., et al. 2005, Informational Bulletin on Variable Stars, 5663, 1

Beloborodov, A. M. 2002, ApJ, 566, L85

Bildsten, L., Chakrabarty, D., Chiu, J., et al. 1997, ApJS, 113, 367

Bulik, T., Gondek-Rosińska, D., Santangelo, A., et al. 2003, A\&A, 404, 1023

Clark, G., Schmidt, G., \& Angel, J. 1975, IAU Circ., 2843

Coburn, W. 2001, Ph.D. Thesis (University of California, San Diego)

Coburn, W., Heindl, W. A., Gruber, D. E., et al. 2001, ApJ, 552, 738

Deeter, J. E., Pravdo, S. H., \& Boynton, P. E. 1981, ApJ, 247, 1003

Dickey, J. M., \& Lockman, F. J. 1990, ARA\&A, 28, 215

Ghosh, P., \& Lamb, F. K. 1979, ApJ, 234, 296

Heindl, W., Coburn, W., Kreykenbohm, I., \& Wilms, J. 2003, ATel, 200

Jahoda, K., Markwardt, C., Radeva, Y., et al. 2006, accepted, ApJS, [arXiv: astroph/0511531]

Kaluzienski, L. 1976, IAU Circ., 2935

Kretschmar, P., Pan, H. C., Kendziorra, E., et al. 1997, A\&A, 325, 623 Kreykenbohm, I. 2004, Ph.D. Thesis (Institut für Astronomie und Astrophysik, Tübingen)

Kreykenbohm, I., Coburn, W., Wilms, J., et al. 2002, A\&A, 395, 129

Kreykenbohm, I., Wilms, J., Coburn, W., et al. 2004, A\&A, 427, 975

Liu, Q., Paradijs, J., \& van den Heuvel, E. 2001, A\&A, 368, 1021

Lomb, N. R. 1976, Ap\&SS, 39, 447

Makishima, K., Ohashi, T., Kawai, N., et al. 1990, PASJ, 42, 295

Mihara, T. 1995, Ph.D. Thesis (University of Tokyo)

Mihara, T., Makishima, K., Ohashi, T., Sakao, T., \& Tashiro, M. 1990, Nature, 346, 250

Morgan, E., Remillard, R., \& Swank, J. 2003, ATel, 199

Muno, M. P., Chakrabarty, D., Galloway, D. K., \& Psaltis, D. 2002, ApJ, 580, 1048

Nagase, F. 1989, PASJ, 41, 1

Nagase, F., Hayakawa, S., Makino, F., Sato, N., \& Makishima, K. 1983, PASJ, 35, 47

Pakull, M., Motch, C., \& Negueruela, I. 2003, ATel, 202

Press, W. H., \& Rybicki, G. B. 1989, ApJ, 338, 277

Rothschild, R. E., Blanco, P. R., Gruber, D. E., et al. 1998, ApJ, 496, 538

Santangelo, A., Del Sordo, S., Segreto, A., et al. 1998, A\&A, 340, L55

Scargle, J. D. 1982, ApJ, 263, 835

Stella, L., White, N., \& Rosner, R. 1986, ApJ, 308, 669

Tanaka, Y. 1986, in IAU Coll. 89: Radiation Hydrodynamics in Stars and Compact Objects, ed. D. Mihalas, \& K. H. Winkler (New York: Springer)

Wegner, W. 1994, MNRAS, 270, 229

Zombeck, M. 1990, Handbook of Astronomy and Astrophysics, 2nd ed. (Cambridge UK: Cambridge University Press) 